\title{
Inpatient Management of COVID-19 Pneumonia: a Practical Approach from the Hospitalist Perspective
}

\author{
Todd S. Cutler, MD, Nell Eisenberg, MD, and Arthur T. Evans, MD, MPH \\ Division of Hospital Medicine, Weill Cornell Medical Center, 525 East 68th Street, Box 130, New York, NY, USA.
}

KEY WORDS: COVID-19; pneumonia; management; hospitalist; health care delivery.

$\mathrm{J}$ Gen Intern Med

DOI: $10.1007 / \mathrm{s} 11606-020-05927-7$

(c) Society of General Internal Medicine 2020

\section{CASE INTRODUCTION}

A 70-year-old man with hypertension and diabetes is admitted to the hospital with cough, shortness of breath, and fevers. The patient's symptoms began 10 days earlier, when he noticed fatigue and subjective fevers. Over the past week, he developed a worsening dry cough and shortness of breath with minimal exertion. In the emergency department, he appears fatigued and is febrile with tachypnea and tachycardia. His oxygen saturation is $84 \%$ on room air and increases to $92 \%$ on 6-1 nasal cannula. His labs are notable for lymphopenia, prerenal azotemia, and hyperglycemia. A nasopharyngeal RTPCR viral swab is positive for SARS-CoV-2. His chest Xray shows diffuse bilateral infiltrates. How should this patient be managed?

\section{BACKGROUND}

On March 11, 2020, the World Health Organization declared the spread of the novel 2019 coronavirus (COVID-19), a pandemic. ${ }^{1}$ Increased admissions for COVID-19 pneumonia ${ }^{2}$, ${ }^{3}$ threaten to overwhelm hospital capacity worldwide. ${ }^{2,4}$ While some patients present with hypoxic respiratory failure ${ }^{5}$ and require emergent intubation, ${ }^{6}$ most patients have progressively worsening respiratory symptoms and require a period of close monitoring. ${ }^{7}$ We have collectively managed over 2400 patients with COVID-19 pneumonia at a single institution at the epicenter of the American outbreak. We have learned valuable lessons on how to manage patients with COVID-19 while conserving resources and minimizing nosocomial spread of infection. In this narrative review, we outline our practice for the management of non-intubated hospitalized patients with COVID-19 pneumonia.

\section{CLINICAL PRESENTATION}

Most patients with COVID-19 have mild to moderate symptoms ${ }^{8}$ and recover without ever encountering a medical provider. This is in part due to widespread guidance from regional Departments of Health that advise patients with mild symptoms to self-isolate. The typical course of COVID-19 pneumonia begins with fever and fatigue followed by a dry cough. ${ }^{9}$ The duration of symptoms can vary, but it is common for patients to describe fatigue and fevers for 7-10 days before their symptoms progress to dyspnea. ${ }^{10}$ Patients frequently have a reduced appetite and, despite data from China describing minimal gastrointestinal symptoms, ${ }^{3}$ many patients complain of diarrhea, abdominal pain, and nausea. Gastrointestinal symptoms may even overshadow respiratory complaints. Some patients have poor appetite along with loss of smell and taste. ${ }^{11}$ Elderly patients or those with underlying cognitive impairment may present with altered mental status. Patients admitted to the hospital generally have moderate to severe respiratory symptoms and hypoxemia at rest. Patients also frequently have comorbidities including obesity, poorly controlled diabetes, hypertension, and coronary artery disease. ${ }^{6,10}$

\section{INITIAL ASSESSMENT AND MANAGEMENT}

Place all COVID-19 pneumonia suspects into contact and droplet isolation immediately on arrival to the hospital. ${ }^{12}$ All patients should be placed on continuous pulse oximetry and wireless telemetry for close central monitoring of oxygen saturation at a nursing station. All patients should be given a surgical face mask and instructed to put it on whenever a health care worker enters the room. Health care workers should review the standard practice recommendations for appropriate donning and doffing of personal protective equipment (PPE) before they enter an isolation room. A trained observer should offer guidance in the donning and doffing process in order to minimize the risk of contamination.

In order to reduce exposure, health care workers need to limit time in the isolation room whenever possible. Most of the admission history can be elicited via telephone, either directly from the patient or from a family member or friend. A detailed social history should include the living situation and the health of household members, functional status in the home environment, and limitations to home isolation that may be present on discharge from the hospital.

Patients must have their advance directives addressed during the first encounter, regardless of their general health. The possibility of intubation must be discussed and advance 
directives clearly documented, as respiratory decompensation can occur rapidly at any point during the admission. When the diagnosis of COVID-19 is confirmed, patients should be informed and educated regarding the expected duration of symptoms which can extend over weeks, hospital isolation procedures, and visitation policy. We begin the conversation about discharge criteria at this time.

We recommend that a single member of the care team enter the isolation room for history and exam. Large team visits are discouraged to reduce health care worker exposure and to conserve PPE. A focused physical exam includes vital signs, an accurate respiratory rate, oxygen saturation, chest inspection for accessory muscle use and work of breathing, and mental status. Daily pulmonary auscultation is of minimal value as findings do not change clinical management and stethoscopes require sterilization in between patients. Patients frequently have diffuse rales that do not appear to correlate with the severity of hypoxemia or work of breathing. If there is concern for an alternative etiology of the patient's shortness of breath, bedside cardiac and lung point-of-care ultrasound ${ }^{13}$ can be used to further evaluate the lungs and the heart. ${ }^{14}$

Patients with COVID-19 pneumonia should remain on isolation throughout their hospitalization. Patients can be cohorted in a single room so as to evaluate multiple patients in sequence, conserve PPE, and minimize repeated patient exposures. Patients should be placed on a single geographical unit to foster close communication with nursing and ancillary staff. Assigning patients to designated COVID-19 teams may reduce the risk of transmission to COVID-19-negative patients. Entries into isolation rooms should be coordinated with nursing and other providers and every patient encounter should serve more than one function such as vital signs assessment, medication administration, phlebotomy, food delivery, and communication.

We assess daily the evolution of oxygen requirements, dyspnea and work of breathing, appetite, mental status, and ability to get out of bed. As with the initial visit, extended conversations at the bedside are avoided. We encourage team members to call patients on their cellular phones to convey results and answer questions. If patients or family members have smart phones or tablets, video chat is a good way to establish rapport and build relationships. An extended care team including counselors, chaplain services, and even trained volunteers can communicate with patients and provide counseling as necessary. Some hospitals may be able to provide patients with smart phones or tablets to use while in isolation.

The volume and frequency of daily blood tests, glucose checks, and medications should be minimized. Most patients with COVID-19 pneumonia outside the ICU require no daily routine laboratory testing, as the white blood count and inflammatory markers such as ESR, CRP, ferritin, d-dimer, and procalcitonin have not been demonstrated to correlate with outcomes independent of the patient's oxygen saturation and work of breathing. All patients should receive medical prophylaxis against deep vein thrombosis unless there is a contraindication. Consultant evaluations should be limited and patient field trips off the ward for radiology testing or procedures should be minimized unless absolutely necessary.

COVID-19 pneumonia does not respond to antibiotics and bacterial pneumonia superinfection is uncommon. When the diagnosis is confirmed and there is no concern for a second infection, antibiotics are unnecessary. Markers of infection such as leukocytosis, elevated procalcitonin, and other inflammatory markers are not helpful in guiding antibiotic use.

\section{SYMPTOM MANAGEMENT}

Acetaminophen can be used on a standing basis for headaches, myalgias, and persistent fever. Opiates such as codeine are effective in relieving cough discomfort and dyspnea. NSAIDs should be avoided given the potential to negatively impair renal blood flow. If diarrhea is profuse or persistent, loperimide can be used to prevent hypovolemia. Patients may require fluid resuscitation until their appetite recovers especially as many patients present with volume depletion. The empiric use of glucocorticoids is not recommended unless there is a separate compelling indication such as a COPD exacerbation. While COVID-19 appears to have a limited bronchoconstrictive effect, patients may feel symptomatic relief with inhaled bronchodilators. In an attempt to avoid the aerosolization of virus particles, we recommend using a metered-dose inhaler in lieu of nebulizer therapy.

Some patients have improved symptoms with awake proning and we have observed improved oxygenation after a prolonged period of pronation. A supportive pillow under the patient's chest and raising the patient's arms above their head can alleviate positional discomfort. A slight degree of reverse Trendelenburg has the same effect. Patients with obesity may notice improvement in the lateral decubitus position although laterality in individual patients will differ depending on which lung is more affected. There is no evidence that proning decreases the need for intubation or shortens the length of hospitalization.

\section{THERAPY}

There is no known treatment for COVID-19 beyond the supportive care of the acute hypoxic respiratory failure. At the time of this writing, all therapies are either investigational or have theoretical benefit without proven efficacy. Drugs with purported anti-viral activity include hydroxychloroquine and remdesivir, ${ }^{15}$ among others. Inflammatory modulators such as the IL-6 inhibitors sarilumab and tocilizumab and the IL-1 inhibitor anakinra may be of benefit in dampening the patient's immune response to the novel coronavirus. ${ }^{16} \mathrm{~A}$ further discussion of investigational drugs for the treatment of COVID-19 pneumonia is beyond the scope of this review. 
There should be consideration to include any patient admitted for the treatment of COVID-19 pneumonia into a clinical trial.

\section{ESCALATION OF CARE/INTUBATION}

We recommend frequent meetings with the critical care and anesthesia teams to review the patients at highest risk of intubation. While there is no cutoff that will apply to every patient, intubation should be considered in patients with decreasing oxygen saturation, escalating oxygen requirements, change in mental status, and increasing work of breathing. We have observed patients tolerate non-rebreather with nasal cannula supplementation for many days before their oxygen requirements subsequently decrease and their symptoms improve.

Once the decision has been made to intubate, it is essential to coordinate with nursing, bed management, critical care, and the intubation team. We recommend a designated negative pressure intubation room on every floor where COVID-19 patients are managed. Patients can be moved to the intensive care unit prior to intubation if their respiratory status is stable for transfer. Given concern for aerosolization of droplets, the use of high-flow nasal cannula or non-invasive positive pressure ventilation should be minimized, unless the patient is in a negative pressure room and a respiratory therapist is present. Use of non-invasive positive pressure ventilation, high-flow nasal cannula, or a bag valve mask should be supervised by critical care and anesthesia.

As intubation is a procedure with high risk for respiratory droplet exposure, only the intubating attending, respiratory therapist, and medication nurse should be present in the room during the procedure. All health care providers should wear full contact, droplet, and respiratory PPE, including an N95 mask. The most experienced provider on the intubation team should manage the patient's airway. The management of intubated patients generally follows the ARDSnet protocol ${ }^{17}$ and is outside the scope of this review.

\section{DISCHARGE PLANNING}

COVID-19 symptoms often last for weeks, and patients are likely to be discharged home before they return to their baseline. Dyspnea and hypoxemia should improve prior to discharge, although neither of these symptoms should be expected to normalize. Fever, cough, diarrhea, and myalgias can be managed at home with acetaminophen and antitussives as needed. The severity of dyspnea with exertion should be assessed prior to discharge.

Recently extubated patients are often fatigued, have poor appetite, and may still require oxygen. Patients are also at risk of reintubation which can depend on their length of intubation, damage to their airway, respiratory strength, and body habitus. They may require many days of close monitoring before their symptoms improve enough to be discharged safely. An early physical therapy assessment and potential referral for acute rehabilitation should be prioritized to improve outcomes in these patients.

As with thresholds for intubation, we have found oxygen saturation cutoffs to inform discharge decisions to be arbitrary. Of greater importance is the general trend and stability of a patient's symptoms and vital signs. Provider comfort with discharge will depend on multiple factors including the patient's functional status and ability to self-monitor for worsening symptoms. For providers' concerns about refractory hypoxemia, it is reasonable to consider oxygen supplementation with nasal cannula on discharge although the value of this is uncertain. Home pulse oximeters and close telephone virtual follow-up visits to assess symptoms can be beneficial.

Patients' living conditions should be assessed in combination with their functional status in order to determine whether self-isolation is feasible and safe. A social worker should help to plan for discharge, including whether the patient will require home care, physical therapy, or assistance with transportation. Undomiciled patients may be eligible for hotels and shelters depending on state and city local resources.

On the day of discharge, we counsel patients in detail about conditions that warrant return to the emergency room. They should return immediately if their breathing worsens. Some home care services may offer remote oxygen saturation monitoring. Lastly, many patients with COVID-19 have poorly controlled chronic conditions such as diabetes that must be addressed prior to discharge since home isolation will preclude close follow-up with a community health care provider.

\section{CASE FOLLOW-UP}

The patient was placed on contact and droplet isolation with 61 nasal cannula and continuous pulse oximetry. His advanced directives and health care proxy information was reviewed. He received 11 of normal saline and antibiotics were not given. $\mathrm{He}$ received acetaminophen with codeine to relieve his fever and cough. He received enoxaparin for deep vein thrombosis prophylaxis and an inpatient insulin regimen for glucose control. He was closely monitored for changes in his oxygen requirements and his work of breathing. On hospital day 2, his oxygen requirements increased and he was placed on a non-rebreather face mask. Critical care was alerted to this change so that a potential intubation could be performed in a controlled manner.

He remained febrile and dyspneic and on hospital day 6, his oxygen requirements and respiratory effort improved along with his appetite. He remained slightly dyspneic with exertion. On hospital day 8 , he was afebrile, did not require oxygen supplementation at rest, and was able to ambulate without severe dyspnea. Prior to discharge, his home isolation plan was reviewed. Lastly, his home medication regimen was adjusted to more effectively manage his diabetes and hypertension. 


\section{CONCLUSION}

We believe that in the current pandemic, it is the role of the health care provider to be continuously assessing patients who are likely to progress to respiratory failure while identifying those who are stable enough to be discharged home. The recommendations here are meant to be pragmatic and aim to meet the multiplicity of challenges hospitals face all over the world including patient outcomes, hospital capacity, human resource limitations, scarcity of PPE, and minimization of nosocomial spread.

Corresponding Author: Todd S. Cutler, MD; Division of Hospital Medicine, Weill Cornell Medical Center, 525 East 68th Street, Box 130, New York, NY 10065, USA (e-mail: Toc9021@med.cornell.edu).

\section{REFERENCES}

1. WHO Director- General's opening remarks at the media briefing on COVID-19: 11 March 2020. 2020;(March). https://www.who.int/dg/ speeches/detail/who-director-general-s-opening-remarks-at-the-mediabriefing-on-covid-19\%2D\%2D-11-march-2020.

2. Grasselli G, Pesenti A, Cecconi M. Critical Care Utilization for the COVID-19 Outbreak in Lombardy, Italy. JAMA. 2020. doi:https://doi. org/10.1001/jama.2020.4031

3. Guan W, Ni Z, Hu Y, et al. Clinical Characteristics of Coronavirus Disease 2019 in China. N Engl J Med. 2020. doi:https://doi.org/10.1056/ nejmoa2002032

4. Yang X, Yu Y, Xu J, et al. Clinical course and outcomes of critically ill patients with SARS-CoV-2 pneumonia in Wuhan, China: a singlecentered, retrospective, observational study. Lancet Respir Med. 2020. doi:https://doi.org/10.1016/S2213-2600(20)30079-5

5. Bhatraju PK, Ghassemieh BJ, Nichols M, et al. Covid-19 in Critically Ill Patients in the Seattle Region - Case Series. N Engl J Med. 2020. doi:https://doi.org/10.1056/NEJMoa2004500
6. Zhou F, Yu T, Du R, et al. Clinical course and risk factors for mortality of adult inpatients with COVID-19 in Wuhan, China: a retrospective cohort study. Lancet. 2020. doi:https://doi.org/10.1016/S0140-6736(20) 30566-3

7. Wang D, Hu B, Hu C, et al. Clinical Characteristics of 138 Hospitalized Patients with 2019 Novel Coronavirus-Infected Pneumonia in Wuhan, China. JAMA - J Am Med Assoc. 2020. doi:https://doi.org/10.1001/ jama.2020.1585

8. Tian S, Hu N, Lou J, et al. Characteristics of COVID-19 infection in Beijing. J Infect. 2020. doi:https://doi.org/10.1016/j.jinf.2020.02.018

9. Zhu N, Zhang $\mathbf{D}$, Wang $\mathbf{W}$, et al. A novel coronavirus from patients with pneumonia in China, 2019. $N$ Engl J Med. 2020. doi:https://doi.org/10. 1056/NEJMoa2001017

10. Huang C, Wang $\mathbf{Y}$, Li X $\mathbf{X}$, et al. Clinical features of patients infected with 2019 novel coronavirus in Wuhan, China. Lancet. 2020. doi:https://doi. org/10.1016/S0140-6736(20)30183-5

11. Bagheri SHR, Asghari AM, Farhadi M, et al. Coincidence of COVID-19 epidemic and olfactory dysfunction outbreak. medRxiv. 2020. https:// doi.org/10.1101/2020.03.23.20041889

12. Chavez S, Long B, Koyfman A, Liang SY. Coronavirus Disease (COVID19): A primer for emergency physicians. Am J Emerg Med. 2020. doi:https://doi.org/10.1016/j.ajem.2020.03.036

13. Peng QY, Wang XT, Zhang LN. Findings of lung ultrasonography of novel corona virus pneumonia during the 2019-2020 epidemic. Intensive Care Med. 2020. doi:https://doi.org/10.1007/s00134-020-05996-6

14. Lichtenstein D. Lung ultrasound in the critically ill. Curr Opin Crit Care. 2014. doi:https://doi.org/10.1097/MCC.0000000000000096

15. Wang $\mathbf{M}$, Cao $\mathbf{R}$, Zhang $\mathbf{L}$, et al. Remdesivir and chloroquine effectively inhibit the recently emerged novel coronavirus (2019-nCoV) in vitro. Cell Res. 2020. doi:https://doi.org/10.1038/s41422-020-0282-0

16. Chen G, Wu D, Guo W, et al. Clinical and immunologic features in severe and moderate Coronavirus Disease 2019. J Clin Invest. 2020. doi:https://doi.org/10.1172/jci137244

17. Alhazzani W, Møller MH, Arabi YM, et al. Surviving Sepsis Campaign: guidelines on the management of critically ill adults with Coronavirus Disease 2019 (COVID-19). Intensive Care Med. 2020. doi:https://doi.org/ $10.1007 / \mathrm{s} 00134-020-06022-5$

Publisher's Note: Springer Nature remains neutral with regard to jurisdictional claims in published maps and institutional affiliations. 